\title{
El Papel Funcionamiento y la Comunicación Familiar en los Síntomas Psicosomáticos
}

\section{The Effect of Family Functioning and Family Communication on Psychosomatic Symptoms}

\author{
Nayeli Rivero-Lazcano \\ Universidad de Deusto, España
}

\author{
Ana Martínez-Pampliega \\ Universidad de Deusto, España
}

\author{
Ioseba Iraurgi \\ I+D+i Psicología Clínica y de la Salud., España
}

\begin{abstract}
Resumen. El objetivo de este estudio ha sido la comprobación del papel de las variables familiares (cohesión, adaptabilidad y satisfacción), en la relación de la comunicación con los síntomas psicosomáticos. Se utilizaron el modelo de los Patrones de Comunicación Familiar (Koerner y Fitzpatrick, 2004) y el Modelo Circumplejo (Olson, 2000) para estudiar la comunicación y el funcionamiento familiar, respectivamente. Para ello se contó con una muestra de 455 participantes de diversas universidades del País Vasco. Los resultados parecen encontrar mayor número de síntomas en los miembros de familias con liderazgo irregular y con roles poco claros y menor número de síntomas físicos cuando se permite la expresión y el intercambio de ideas, pensamientos y sentimientos. Con este estudio se abre una nueva línea de investigación que permitirá profundizar en la comprensión de los síntomas psicosomáticos.

Palabras clave: Comunicación familiar; funcionamiento familiar; satisfacción; síntomas psicosomáticos.
\end{abstract}

\begin{abstract}
The goal of this study was to test the effect of family variables (cohesion, adaptability and satisfaction) on the relationship between communication and psychosomatic symptoms. The Family Communication Patterns Model (Koerner \& Fitzpatrick, 2004) and the Circumplex Model (Olson, 2000) were used to study family communication and family functioning. The sample consisted of 455 university students in the Basque Country. The results emphasize that there are more symptoms in members of families with irregular leadership and with roles that are not clear, whereas those families with an open expression of ideas, thoughts and feelings, tend to have fewer symptoms. These results open a new line of research that will help to verify the results presented and to have a better comprehension of the psychosomatic symptoms.

Keywords: family communication; family functioning; satisfaction; psychosomatic symptoms.
\end{abstract}

Existen múltiples factores involucrados en la aparición y mantenimiento de los síntomas psicosomáticos. Entre ellos se pueden mencionar factores psicológicos como el optimismo, la ansiedad y la forma de enfrentar el estrés (Davidsdottir, 2006; Holahan y

La correspondencia sobre este artículo debe enviarse a la segunda autora a la Universidad de Deusto. Facultad de Psicología. Avda. Universidades s/n, 48007 Bilbao. E-mail: martinez.pampliega@ deusto.es
Moos, 1986), el estilo de atribución (Peterson, Vaillant y Seligman, 1988), el estilo de vida (Davidsdottir, 2006), las diferencias de género (Barsky, Peekna y Borus, 2001; Tamada, 2005) y factores psicosociales, como las interacciones familiares (Segrin, 2006), específicamente la comunicación, el funcionamiento familiar (Ochs et al., 2005; Sayre, 2001; Terre y Ghiselli, 1997) y la satisfacción familiar (Horner, 2001; Segrin, 2006). 
Desde hace décadas, ha tenido especial relevancia el estudio del papel de la familia en la explicación de los síntomas somáticos, teniendo gran reconocimiento la perspectiva sistémica. Por ejemplo, Minuchin, Rosman, Baker y Liebman (1978), resaltan que además de la vulnerabilidad de la persona, las características transaccionales en la familia (apego excesivo, sobreprotección, rigidez y evitación del conflicto) y el rol que juega el hijo sintomático tienen un papel en la presencia de los síntomas psicosomáticos. Por otro lado, las investigaciones del grupo dirigido por Onnis (1997) han permitido describir una tipología de familia psicosomática que corresponde a la descrita por Minuchin et al., (1978): familias muy complicadas, con fronteras débilmente delimitadas y con una tendencia a la intrusión en los espacios físicos y psicoemocionales de sus miembros. Presentan un grado muy bajo de tolerancia hacia las tensiones conflictivas, lo que impide cualquier manifestación de desacuerdo. Los vínculos son tan complicados que entorpecen los procesos de individualidad y autonomía.

En investigaciones más recientes, se ha observado la relación entre los patrones de interacción familiar y algunas enfermedades (Sayre, 2001) o diversos dolores (Ochs et al., 2005); además, las personas que utilizan habilidades positivas de comunicación y que mantienen relaciones familiares de armonía son más saludables que aquéllas con relaciones problemáticas (Segrin, 2006). Los miembros de las familias que con mayor frecuencia manifiestan enfermedades somáticas, provienen de familias con interacciones en las que predomina una comunicación evitativa y negativa (Sayre, 2001).

También se ha observado la relación entre el bienestar y la insatisfacción familiar. Por ejemplo, una mayor satisfacción con el matrimonio parece disminuir el impacto del estrés entre parejas de mujeres con cáncer de pecho y se relaciona con una mejor salud mental: menores niveles de depresión, afecto negativo y estrés percibido y mayores niveles de afecto positivo y de salud mental global (Segrin, Badger, Sieger, Meek, y Lopez, 2006). Por el contrario, tener relaciones íntimas insatisfactorias puede predecir futuras enfermedades (Horner, 2001; Segrin, 2006).
Las investigaciones nombradas destacan dimensiones de la comunicación y del funcionamiento familiar, lo que indica que son dos variables fundamentales en el estudio de los síntomas psicosomáticos. Por ello, consideramos relevante el estudio de la relación de los patrones de interacción y comunicación familiar, por un lado y los síntomas psicosomáticos por el otro, partiendo de modelos de estudio con suficiente respaldo empírico y solidez teórica. A juzgar por las investigaciones más recientes en el ámbito del bienes$\operatorname{tar}$ (Kouneski, 2000; Schrodt y Ledbetter, 2007), destacamos el Modelo Circumplejo (Olson, 2000) y el modelo de los Patrones de Comunicación Familiar (Koerner y Fitzpatrick, 2002a), como los modelos que más corresponden a estos criterios.

Las variables del funcionamiento familiar en el modelo circumplejo se encuentran agrupadas en tres dimensiones centrales: cohesión, adaptabilidad y comunicación. La cohesión familiar se define como "el vínculo emocional que tienen los miembros de la familia entre sî" (Olson, Sprenkle y Russell, 1979; p. 9). La adaptabilidad familiar se define como "la cantidad de cambio en el liderazgo, en las relaciones de roles y en las reglas dentro de las relaciones de un sistema marital o familiar" (Olson y Gorall, 2003, p. 519). La comunicación es considerada como facilitadora del cambio en las otras dos dimensiones. Las habilidades de comunicación positivas (empatía, escucha reflexiva, frases de apoyo, mensajes claros y congruentes y habilidades efectivas de resolución de problemas) permiten a las familias compartir entre ellos sus necesidades en relación con la cohesión y la adaptabilidad.

El modelo de los Patrones de Comunicación Familiar describe las tendencias de las familias a desarrollar modos de comunicación estables y predecibles (Koerner y Fitzpatrick, 2004). Se definen según la orientación a la conversación y la orientación a la conformidad (Koerner y Fitzpatrick, 2002b), que son centrales en el funcionamiento familiar. La orientación a la conversación es el grado en que las familias crean un clima que anima a la participación con libertad en las interacciones. Provee de herramientas necesarias para enfrentar los eventos estresantes de la vida $\mathrm{y}$, en concordancia con lo encontrado por Schrodt y Ledbetter (2007), podría tener una relación inversa con los síntomas 
psicosomáticos: ambientes que promueven apertura y expresión, facilitan un desarrollo saludable en los hijos.

La orientación a la conformidad se refiere al grado en el que la comunicación familiar propicia la homogeneidad en actitudes, valores y creencias; no implica más cohesión, sino que tiene claras características coercitivas en la mayoría de las familias.

Otra dimensión estudiada es la satisfacción familiar, que ha demostrado relacionarse con ambas dimensiones; sin embargo, en la orientación a la conformidad las correlaciones fueron más bien bajas, sobre todo en la dimensión que evalúa la obediencia, lo cual puede deberse a que la satisfacción con la vida familiar tiene poca conexión con obedecer a los padres o no (Rivero y Martínez-Pampliega, 2010). Otros estudios han demostrado la relación entre la comunicación positiva y la satisfacción en la relación marital (Flora y Segrin, 2000, citado en Segrin, 2006).

La revisión anterior parece indicar que la forma en que las variables del funcionamiento familiar se relacionan con los síntomas psicosomáticos y cómo pueden condicionar la relación de la comunicación con los síntomas no está suficientemente clarificada. Por ello, el objetivo de este estudio ha sido la comprobación del impacto de las variables familiares cohesión y adaptabilidad, (y complementariamente la mediación de la satisfacción), en la relación entre la comunicación y los síntomas psicosomáticos.

La orientación comunicativa de las familias, condicionada por el funcionamiento familiar, se vincula con los síntomas psicosomáticos de los hijos, pero siempre mediada por la satisfacción familiar.

\section{Método}

\section{Participantes}

La población elegida fueron alumnos universitarios. Se trató de una muestra incidental que tuvo en cuenta la inclusión de estudiantes de diferentes cursos, titulaciones y universidades del País Vasco. Se solicitó la participación voluntaria y confidencial. Participaron un total de 455 sujetos aplicando dos criterios de homogenización: (a) el intervalo de edad universitaria, tomando en cuenta la media de edad y +/-1 desviación típica; (b) convivencia familiar, considerando a quienes viven con sus padres toda la semana o al menos durante el fin de semana. Una vez aplicados dichos criterios, y elminando los casos que no contestaron todas las preguntas, se contó con una muestra final de 429 participantes. El rango de edad estuvo situado entre 18 y 25 años con una media de 20.5 años y estuvo formada mayoritariamente por mujeres $(73.6 \%)$.

\section{Instrumentos}

- RFCP (Koerner y Fitzpatrick, 2002a): Esta escala evalúa los patrones de comunicación familiar. La versión española cuenta con dos escalas para la Orientación a la conversación (aceptación de la diferencia, con 4 ítems y expresión de ideas, con 7 ítems) y dos para la orientación a la conformidad (rechazo de la diferencia, con 2 ítems y ánimo a la obediencia con 3 ítems), a las que se responde con una escala tipo likert de 5 opciones, según el grado de acuerdo. Las escalas probaron ser válidas y razonablemente adecuados en su fiabilidad (alpha: entre 89 y .76.) (Rivero y MartínezPampliega, 2010) y se realizó una suma simple para la variable global.

- FACES IV (Olson, Gorall y Tiesel, 2006): La versión española cuenta con 24 ítems divididos en dos escalas equilibradas (cohesión y adaptabilidad) y cuatro escalas desequilibradas que miden los extremos superiores e inferiores de la cohesión (apego y desapego) y de la adaptabilidad (caos y rigidez), con 4 ítems cada escala. Todas ellas se responden con una escala tipo likert de 5 opciones y las variables globales se realizaron con una suma simple. Estas escalas probaron ser válidas y los valores de fiabilidad, cercanos a la adecuación (alpha: entre .77 y .50) (Rivero, Martínez-Pampliega y Olson, 2010).

- FSS (Family Satisfaction Scale) Escala de Satisfacción Familiar (Olson, Stewart y Wilson, 1990). Evalúa el grado de satisfacción con aspectos relacionados con la cohe- 
sión y la adaptabilidad familiar, demostrando buenos índices de fiabilidad (alpha de Cronbach de .92, Sanz, Iraurgi y MartínezPampliega, 2002). Cuenta con 10 ítems y se responde con una escala tipo likert de 5 opciones, haciendo una suma simple para las variables resumen.

- YASR: se utilizó la subescala "quejas somáticas" del Autoinforme del Comportamiento de Jóvenes de 18 - 30 años de Achenbach (1991), que consta de 10 síntomas; sus ítems se centran en problemas o molestias sin causa médica. Se responde teniendo en cuenta el grado de acuerdo con una de tres opciones de respuesta (no es cierto; algo, algunas veces cierto; cierto muy a menudo o bastante a menudo) según los síntomas se hayan presentado o no en los últimos seis meses. Tiene una adecuada fiabilidad de .79 en estudios realizados con poblaciones similares a la de este estudio (Cosgaya, Nolte, Martinez-Pampliega, Sanz, y Iraurgi, 2008).

\section{Estrategia de análisis}

Con el fin de favorecer su comparabilidad, las puntuaciones de los instrumentos se convirtieron a una escala decimal. De este modo, las características evaluadas se interpretaron utilizando una valoración ampliamente conocida (cero equivale a ausencia de la característica y diez al máximo posible de expresión). Para la descripción de la muestra se utilizaron las medidas de tendencia central (Media $-M$ ) y dispersión (Desviación típica - DT). Se analizó la fiabilidad de las escalas utilizadas a través del coeficiente alpha de Cronbach (a), ofreciendo una estimación de la consistencia interna de las mismas. También se examinó la asimetría y la curtosis de cada variable y se realizó la prueba de normalidad de Kolmogorov- Smirnov.

El primer paso fue la comprobación de un modelo estructural considerando las variables latentes indicadas. Debido que el tamaño de la muestra no fue suficiente para el número de variables del modelo, se optó por comprobar una versión simplificada del mismo. Para ello se realizaron los siguientes pasos, previos a la comprobación del modelo:
1. Se realizó un análisis factorial confirmatorio con los ítems correspondientes al instrumento de funcionamiento familiar, comprobando la saturación de los 4 ítems por cada una de las 6 dimensiones (modelo ya publicado, Rivero, Martínez-Pampliega y Olson, 2010) y su confluencia a su vez, en una variable latente: funcionamiento familiar.

2. Se realizó un análisis factorial confirmatorio con los ítems del instrumento de comunicación, considerando dos variables latentes: Orientación a la conversación y orientación a la conformidad.

En todos los casos, para evaluar el nivel de bondad de ajuste del modelo hipotetizado, se utilizaron diversos índices entre los que se encuentra el ji-cuadrado $\left(\chi^{2}\right)$, que indica la probabilidad de que la divergencia entre la matriz de varianzas y covarianzas muestrales y la generada a partir del modelo hipotetizado sean debidas al azar. Dado que el $\chi^{2}$ es muy sensible a las variaciones del tamaño de la muestra se emplearon medidas adicionales de la bondad de ajuste del modelo, como el error de aproximación cuadrático medio (RMSEA), considerando aceptables valores menores que .06; el Bollen Fit Index (IFI), el índice de ajuste comparado (CFI) y el índice de bondad del ajuste $(G F I)$, cuyos valores deben ser superiores a 0.95. Como método de estimación se utilizó el de máxima verosimilitud. Para interpretar los resultados se eligió un nivel de significación de $p<0.05$ para un intervalo de confianza del $95 \%$. Los análisis se realizaron con el programa EQS.

\section{Resultados}

Se inició realizando análisis descriptivos de las variables consideradas. La población estudiada mostró un adecuado funcionamiento familiar observado en las escalas centrales (cohesión $M=7.33, D . T$. = .76 ; adaptabilidad $M=6.04, D . T .=.79)$ y en los niveles bajos de las escalas extremas (apego $M=2.78$, D.T. $=.66$; desapego $M=1.87, D . T .=.77$; rigidez $M$ $=3.13, D . T .=.76$ y caos $M=2.05, D . T .=.73)$, con un notable nivel de satisfacción familiar $(M=7.36$, $D . T .=.76)$ (tabla 1). La asimetría de las escalas centrales del funcionamiento familiar fue negativa, indi- 
Tabla 1. Análisis de fiabilidad y características descriptivas de las dimensiones estudiadas

\begin{tabular}{|c|c|c|c|c|c|c|c|c|c|}
\hline & $\mathrm{N}^{o}$ ítems & Fiabilidad & $\begin{array}{c}\mathrm{N}^{\mathrm{o}} \\
\text { sujetos }\end{array}$ & $\begin{array}{c}\text { Media } \\
\text { escala } \\
\text { bruta }\end{array}$ & $\begin{array}{c}\text { Media } \\
\text { escala } \\
\text { decimal }\end{array}$ & $\begin{array}{l}\text { Desv. } \\
\text { Típica }\end{array}$ & As & Curt & $\begin{array}{c}\text { Estadístico } \\
\text { Kolmogorov- } \\
\text { Smirnov }\end{array}$ \\
\hline \multicolumn{10}{|l|}{ FACES IV } \\
\hline Cohesión & 4 & .77 & 455 & 3.94 & 7.33 & .76 & -1.11 & 1.75 & $.16^{* *}$ \\
\hline Adaptabilidad & 4 & .65 & 455 & 3.42 & 6.04 & .79 & -.59 & .193 & $.14^{* *}$ \\
\hline Apego & 4 & .51 & 455 & 2.40 & 2.78 & .66 & .29 & -.21 & $.11 * *$ \\
\hline Desapego & 4 & .74 & 455 & 1.94 & 1.87 & .77 & 1.04 & 1.18 & $.14 * *$ \\
\hline Rigidez & 4 & .70 & 455 & 2.57 & 3.13 & .76 & .185 & -.19 & $.08 * *$ \\
\hline Caos & 4 & .65 & 455 & 2.03 & 2.05 & .73 & 1.08 & 1.70 & $.13^{* *}$ \\
\hline \multicolumn{10}{|l|}{ RFCP } \\
\hline Acept. Diferencia & 4 & .81 & 451 & 3.33 & 4.63 & .88 & -.34 & .12 & $.08 * *$ \\
\hline Expresión de ideas & 7 & .89 & 450 & 3.56 & 5.65 & .87 & -.70 & .40 & $.10 * *$ \\
\hline Rech. Diferencia & 2 & .76 & 443 & 2.45 & 2.90 & 1.07 & .47 & -.55 & $.17 * *$ \\
\hline Obediencia & 3 & .73 & 444 & 3.34 & 4.66 & .91 & -.30 & -.07 & $.10^{* *}$ \\
\hline Satisfacción Familiar & 10 & .91 & 453 & 3.51 & 7.36 & .76 & -.99 & 1.45 & $.10 * *$ \\
\hline YASR/Quejas somáticas & 10 & .72 & 433 & 1.35 & 1.65 & .32 & 1.74 & 4.38 & $.16^{* *}$ \\
\hline
\end{tabular}

Nota. ${ }^{* *} p<.001$. As= Asimetría; Curt= Curtosis

cando una mayor agrupación de los valores altos de estas variables; las escalas extremas presentaron asimetría positiva, mostrando mayor agrupación en los valores bajos. Esto era esperable, ya que la medición se realizó con familias no clínicas. En el caso de la curtosis, sólo en las escalas de apego y rigidez fue negativa, indicando la presencia de muchos casos en los extremos. La asimetría de la satisfacción familiar fue negativa y la curtosis, positiva.

En la comunicación familiar ${ }^{1}$, se presentaron niveles adecuados en la aceptación de la diferencia $(M=4.63, D . T .=.88)$ y en la expresión de ideas $(M$ $=5.65, D . T .=.87)$ y bajos en el rechazo a la diferencia $(M=2.90, D . T .=1.07)$. En la obediencia $(M=$ $4.66, D . T .=.91)$, la media tuvo un nivel moderado, siendo éste más alto de lo esperado. La aceptación de la diferencia, la expresión de ideas y la obediencia tuvieron asimetría negativa, indicando puntuaciones altas. Sólo el rechazo de la diferencia tuvo asimetría positiva. En el caso de la curtosis, en el rechazo a la diferencia y la obediencia fue negativa, aunque en este segundo caso, muy baja.

En la variable "síntomas somáticos" la asimetría fue positiva, indicando valores bajos, y la curtosis positiva. En cuanto a las frecuencias, los participan-

${ }^{1}$ Parte de estos resultados fueron presentados en el VI Congreso de la Asociación Española para la Investigación y Desarrollo de la Terapia Familiar, sobre "Terapia Familiar y Salud", celebrado en Mayo de 2009 en Santander, España. tes presentaron un nivel bajo de sintomatología física $(M=1.65, D . T .=.32)$. En todos los casos, el test de normalidad fue significativo, lo que sugiere una violación de este supuesto. Sin embargo es común que esto suceda en muestras grandes (por ejemplo, mayores que 30) y no debe suponer problemas en los análisis posteriores (Pallant, 2001).

El primer paso para la comprobación del modelo planteado, fue el analisis del instrumento de funcionamiento familiar. En un estudio previo (Rivero, Martínez-Pampliega y Olson, 2010) se comprobó la saturación de los 4 ítems de cada subescala de la cohesión (cohesión central, apego y desapego) y de cada subescala de la adaptabilidad (adaptabilidad central, rigidez y caos), resultando en dos dimensiones latentes (cohesión y adaptaiblidad) con buenos índices de ajuste: $\chi^{2}(237 N=455)=463.337$, RMSEA $=.046 ; \mathrm{CFI}=.97 ; \mathrm{GFI}=.92$. Solamente la dimensión de rigidez no demostró un buen ajuste con el resto del modelo. Paralelamente se comprobó el modelo con una variable latente de funcionamiento familiar: $\mathrm{X}^{2}$ (244): 486.35, $\mathrm{p}<.001$; GFI: .91, CFI: .91, RMSEA: 0.048). De nuevo, la rigidez no mostró un buen ajuste al modelo confirmatorio final, así que se decidió eliminar. Como ambos modelos dieron buenos resultados, en aras de la parsimonia se optó por la consideración de las 6 dimensiones y su confluencia en una única variable latente. 
El segundo paso, fue la comprobación del modelo de comunicación. En este caso, el modelo fue adecuado tomando en cuenta, según los planteamientos teóricos, dos variables latentes: orientación a la conversación y orientación a la conformidad: $\chi^{2}(99)=$ 234,33, p > 0.001; GFI: .93, CFI: .96; RMSEA: 0.057 (0.047 - 0.066), cuya covarianza fue de -.56.

Como último paso, se realizó un análisis estructural para poder comprobar la adecuación de la relación del funcionamiento familiar con la comunicación (orientación a la conversación y orientación a la conformidad), en la explicación de los síntomas psicosomáticos, con la satisfacción como variable mediadora. En el caso del ajuste del modelo y como se puede observar en la figura 1, éste fue, sólo razonablemente adecuado. El valor RMSEA indicó un ajuste cercano al aceptable (.089), al igual que los índices de la bondad del mismo $(\mathrm{CFI}=0.84, \mathrm{GFI}=$ $0.86, \mathrm{IFI}=.86$ ). A pesar de que el ajuste del modelo es sólo cercano a lo aceptable, se presentará a continuación su interpretación debido a que permite tener un acercamiento a la explicación de los síntomas psicosomáticos y abrir futuras líneas de continuidad. En primer lugar, las dimensiones del funcionamiento y comunicación familiar tienen influencia en la satisfacción. Este impacto fue positivo y alto en el caso del funcionamiento familiar (.89) y de orientación a la conversación (.79) y negativo y bajo con la orientación a la conformidad (-.18). Es decir, de acuerdo con lo esperado, un funcionamiento familiar central, en el que hay un equilibrio en el nivel de cercanía de las personas (cohesión) y en su nivel de adaptación a los cambios (adaptabilidad) se asocian con mayor satisfacción con la vida familiar. Lo mismo sucede en el caso de las familias que están abiertas a las conversaciones y en las que los miembros sienten libertad para expresarse. Contrariamente, aquéllas familias en las que sus miembros tienden a una mayor homogeneidad en sus creencias, valores y actitudes, la satisfacción es menor. Sin embargo, este último resultado habrá que tomarlo con reserva, debido a que el valor fue bajo.

Figura 1. Modelo explicativo de los síntomas psicosomáticos

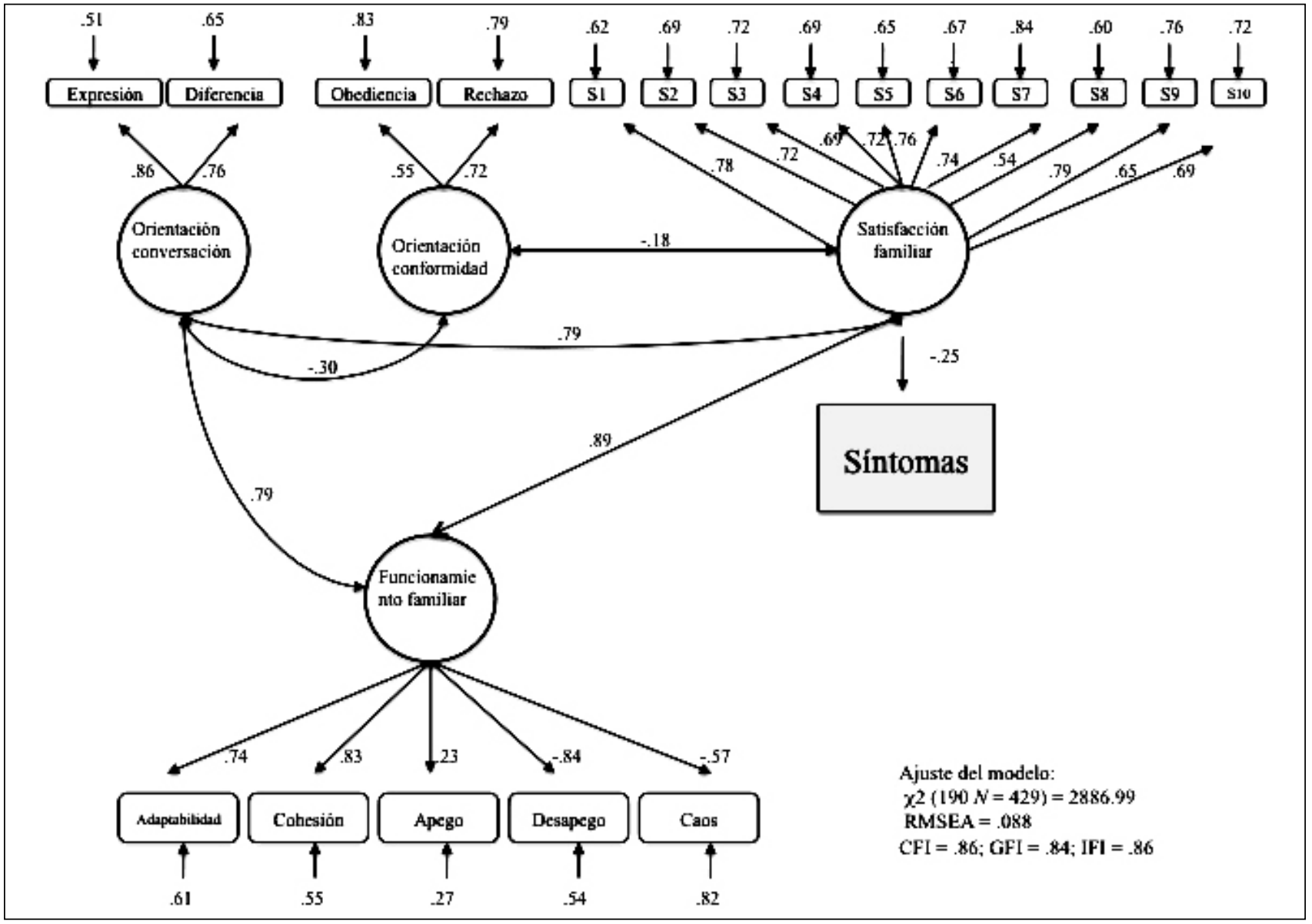


Por otro lado, el grado de satisfacción indicado por los hijos, presenta un efecto negativo en los síntomas.

Lo que el modelo refleja, a modo de conclusión, es que el funcionamiento y la comunicación familiar tienen un efecto en los síntomas psicosomáticos, tomando en cuenta el papel de la satisfacción que los hijos sienten en sus familias; sin embargo, cabe aclarar que el nivel de ajuste del modelo no fue suficientemente adecuado. Una posible razón de lo anterior, es la necesidad de incluir otras variables, además del funcionamiento y la comunicación familiar en la explicación de los síntomas en los hijos. Como se mencionó al inicio, los síntomas psicosomáticos se han relacionado con variables psicológicas, estrés y diferencias de género.

\section{Discusión}

En este estudio el objetivo ha sido la comprobación del papel conjunto de las variables familiares (cohesión, adaptabilidad, satisfacción), en el estudio de la relación entre la comunicación y los síntomas psicosomáticos. La influencia de los factores psicosociales en las enfermedades ha sido estudiada previamente dentro del campo de estudio de la psicosomática (Fava y Sonino, 2005), y desde un punto de vista biopsicosocial, reconociendo la influencia de múltiples elementos en la salud y en la enfermedad, incluidos factores sociales (Taylor, 1991). Esta investigación se ha enfocado en la familia.

El primer paso fue realizar la descripción de la muestra en las variables estudiadas. Tal y como se esperaba, se encontró que la mayoría de las familias tienen un funcionamiento familiar central y una comunicación abierta y expresiva. También se concluyó que los participantes se sienten satisfechos con su nivel de cohesión y adaptabilidad. Sin embargo, se pudo observar que los participantes perciben la rigidez, el apego y la obediencia como algo común en las familias y no como un funcionamiento extremo. Lo anterior puede indicar que los hijos tienden a conformarse y a obedecer las normas de los padres y son éstos quienes toman las decisiones. Esto puede relacionarse con la frecuencia de la dimensión de rigidez, que también evalúa la obediencia y las normas. La sintomatología fue baja debido a que se trata de una población no clínica.

$\mathrm{Al}$ analizar el modelo propuesto, que incluye las variables de la comunicación (Orientación a la conversación y orientación a la conformidad), el funcionamiento familiar y la satisfacción, se observó que el ajuste, aunque bajo, indica que puede ser utilizado como planteamiento inicial en la comprensión de los síntomas psicosomáticos.

Resulta interesante observar la forma en que la comunicación y el funcionamiento familiar interactúan. Estos resultados dejan claro que aparentemente ambas variables están en un mismo nivel en su impacto en los síntomas psicosomáticos. La variable que puede estar marcando la diferencia en esta relación es la satisfacción. Es decir, dependiendo del grado de satisfacción de los miembros de la familia con su funcionamiento familiar (incluyendo la comunicación) se tendrán más o menos síntomas psicosomáticos. Lo que significa que, en ciertas culturas un tipo de funcionamiento puede ser disfuncional, pero en otras no. Esto nos confirma la importancia de tomar en cuenta la "cultura" en las conclusiones de los estudios, cuyo impacto en el funcionamiento familiar ya está siendo considerado, como por ejemplo al analizar la forma en que los valores, actitudes, deseos o creencias en el medio sociocultural en el que viven los padres e hijos explican las normas de las relaciones y su impacto en el bienestar de los miembros de las familias (García y Peralbo, 2000).

Como se puede observar, la comunicación y el funcionamiento se relacionan con los síntomas somáticos. No obstante, y como ya se mencionó, los resultados fueron más bien bajos; quizá pueda deberse al empleo de una muestra no clínica, en la cual la presencia de síntomas somáticos o tipologías familiares extremas no es tan frecuente. Por otro lado, existen otras muchas variables que se han relacionado con la explicación de los síntomas, como por ejemplo, la forma de afrontar el estrés (Zikmund, 1992; Coughlin Della Selva, 2006) y el conflicto familiar (McVey, 2002). Esto lleva a la consideración de que, en estudios posteriores, otras variables pueden ser añadidas al modelo de forma que la explicación que se obtenga explique mayor varianza de la sintomatología. 
Queda mucho camino por avanzar en la investigación de la comunicación familiar y su relación con consecuencias en los miembros de la familia, espe- cíficamente los síntomas somáticos, utilizando como marco de referencia un punto de vista biopsicosocial.

\section{Extended Summary}

The goal of this study was to test the effect of family variables (cohesion, adaptability and satisfaction) on the relationship between communication and psychosomatic symptoms.

Psychosomatic symptoms can be explained by a number of different factors, such as psychological factors (anxiety, stress coping and optimism) (Davidsdottir, 2006; Holahan \& Moos, 1986), attribution style (Peterson, Vaillant \& Seligman, 1988), lifestyle (Davidsdottir, 2006), gender differences (Barsky, Peekna \& Borus, 2001; Tamada, 2005) and psychosocial factors, such as family interactions (Segrin, 2006), specifically family communication and family functioning (Ochs et al., 2005; Sayre, 2001; Terre \& Ghiselli, 1997) and family satisfaction (Horner, 2001; Segrin, 2006).

Recent research has shown the relationship between family interaction patterns and some illnesses (Sayre, 2001), or some pains (Ochs et al., 2005). For example, family members with more somatic illnesses, come from families with avoidant and negative communication (Sayre, 2001).

The relationship between well-being and family dissatisfaction has been explored too, and it has been found that intimate relationships that are not satisfactory can predict future illnesses (Horner, 2001; Segrin, 2006).

In order to study family functioning and communication, two of the most relevant models were used. The Family Communication Patterns Model (Koerner \& Fitzpatrick, 2004) and the Circumplex Model (Olson, 2000).

The Family Communication Patterns Model describes the way in which families develop communications patterns that are stable and predictable. It has two main variables: Conversation orientation, which describes "the degree to which families create a climate in which all family members are encouraged to participate in unrestrained interactions about a wide array of topics" (Koerner \& Fitzpatrick, 2002 b, p. 85); conformity orientation, referring to "the degree to which family communication stresses a climate of homogeneity of attitudes, values, and beliefs" (Koerner \& Fitzpatrick, 2002b, p. 85).

The Circumplex Model, on the other hand, establishes 3 dimensions that explain family functioning: cohesion, adaptability and communication. Family cohesion refers to the emotional bonding that family members have between each other (Olson, Sprenke and Russell, 1979). Family adaptability is defined as the "amount of change in its leadership, role relationships and relationship rules" (Olson y Gorall, 2003, p. 519). Communication is the third dimension and it is considered as a facilitating dimension.

The revision of literature seems to show that the relationship between family functioning and psychosomatic symptoms is not clear. For this reason, the aim of this study is to test the impact of the family functioning variables (cohesion and adaptability), on the relationship between the communication and psychosomatic symptoms.

\section{Method}

\section{Participants}

The sample consisted of 455 university students in the Basque Country. Two criteria where used in order to homogenize the sample: (a) the university age interval (using the age average and $+/-1$ standard deviation; (b) living at home, during the week or only the weekends. Using these two criteria and after eliminating the cases which did not answer all the questions, the final sample consisted of 429 participants. The ages were between 18 and 25 years old, with an average of 20.5 years, and it was mainly made up of women $(73.6 \%)$.

\section{Instruments}

- RFCP (Koerner and Fitzpatrick, 2002a): This scale evaluates family communication patterns. 
The Spanish version has two scales for conversation orientation (acceptance o difference and expression of ideas) and two for conformity orientation (rejection of difference and obedience). The scales prove to be valid with a reasonable level of reliability (alpha: between .89 y .76.) (Rivero \& Martínez-Pampliega, 2010).

- FACES IV (Olson, Gorall y Tiesel, 2006): The Spanish version has 24 items divided in two balanced scales (cohesion and adaptability) and four unbalanced scales which measure the extremes of cohesion (enmeshed and disengaged) and of adaptability (chaos and rigidity), with 4 items each. The scales prove to be valid and have a suitable level of reliability (alpha: entre .77 y .50) (Rivero, Martínez-Pampliega \& Olson, 2010).

- Family Satisfaction Scale (Olson, Stewart y Wilson, 1990). This scale evaluates the degree of satisfaction with family functioning. It has good reliability (Cronbach's alpha $=.92$, Sanz, Iraurgi y Martínez-Pampliega, 2002).

- YASR: The subscale of somatic complaints from the Youth self report (Achenbach, 1991) was used. This has 10 items about physical problems without medical cause. It has a good reliability of .79 in similar samples (Cosgaya, Nolte, Martinez-Pampliega, Sanz, \& Iraurgi, 2008).

\section{Strategy of analysis}

A description of the sample was made with central tendency measures; the reliability of the scales was reported by the Cronbach alpha, as well as the Komogorov-Smirnov normality test.

The structural model of the latent variables was proved. However, the size of the sample was not big enough for the number of variables of the model. For this reason, a simplified version of the model was tested, using the following next steps:

1. Confirmatory factor analysis of the family functioning instrument, according to a previously-published model (Rivero, MartínezPampliega y Olson, 2010). This gave one latent variable: family functioning.
2. Confirmatory factor analysis of the family communication instrument, considering two latent variables: conversation and conformity orientation.

In order to assess the degree of goodness of fit of the hypothesized model, we used several indexes, amongst others the chi-square $\left(\chi^{2}\right)$. Given that it is very sensitive to variations in the size of the sample, additional measures of goodness of fit were used, such as the root mean square error of approximation (RMSEA), for which values below .06 are considered acceptable. We also used the incremental fit index (IFI), the comparative fit index (CFI) and the goodness of fit index (GFI), for which values of .95 or over reflect a good fit.

\section{Results}

The results of the descriptive analysis showed that family functioning was balanced, and low in the extreme scales. In the communication scales there were adequate levels of acceptance of difference and expression of ideas. In obedience, the average was higher than expected. The participants presented a low level of psychosomatic symptoms.

The first step was to analyze the model. In a previous study (Rivero, Martínez-Pampliega \& Olson, 2010), the model of family functioning, with 4 items in each subscale was proved with good adjustment indexes $\chi^{2}(237 \mathrm{~N}=455)=463.337$, RMSEA $=.046$; $\mathrm{CFI}=.97$; GFI $=.92$. Parallel to this, the model with one latent variable of family functioning was proved: $\chi^{2}(244): 486,35$, p < 0.001; GFI: .91, CFI:. 91, RMSEA: 0.048. In both cases, the rigid scale did not have a good adjustment. As both models had good results, it was decided to keep the model of one latent variable, in accordance with the principles of parsimony.

The second step was to prove the communication model. In this case, two latent variables were considered, according to the theoretical model: conversation and conformity orientation: $\chi^{2}(99)=234,33$, p > 0.001; GFI: .93, CFI: .96; RMSEA: 0.057 (0.047 - 0.066).

The last step was the structural analysis in order to prove the model of family functioning, family 
communication and symptoms, with family satisfaction as mediating variable. In this case, the fit of the model was reasonable adequate. The RMSEA value was close to adequacy (.089), as well as the other fit indexes $(\mathrm{CFI}=0.84, \mathrm{GFI}=0.86, \mathrm{IFI}=.86)$. Despite the fact that the model was only close to an acceptable fit, the interpretation would be made, because it allowed us to begin to explain psychosomatic symptoms and opens up future lines of research.

Firstly, family functioning and family communication are related with family satisfaction. This means that central types of family functioning, in which a balanced level of closeness and a good adaptation to changes is shown, are associated with greater family satisfaction. The same pattern was found in families that are open to conversations and in which members feel free to express themselves. The degree of satisfaction, however, has a negative effect on symptoms.

\section{Discussion}

What the model shows is that family functioning and family communication have an effect on psychosomatic symptoms, taking in consideration the satisfaction that children feel with their families.

It is interesting to analyze how family functioning and family communication interact. These results show clearly that both variables are on the same level in the explanation of psychosomatic symptoms. The variable that makes the difference is family satisfaction. This means that the number of psychosomatic symptoms depends on how satisfied family members are with family functioning (including family communication). This means that in some cultures, one kind of family functioning can be dysfunctional, but in others not. That confirms how important it is to take into consideration the "culture" variable in research.

One limitation of this study was that the fit of the model was only close to adequate. One possible explanation is that a non-clinical sample was used in which psychosomatic symptoms or extreme family functioning is not frequent. In addition, there are other variables that have been related with symptoms, like the way of coping with stress (Coughlin
Della Selva, 2006; Zikmund, 1992) and family conflict (McVey, 2002). This means that other variables can be added to the model that explains psychosomatic symptoms.

There is still a long way to go in the research of family functioning and outcomes in the family, and, specifically psychosomatic symptoms.

\section{Referencias}

Achenbach, T. M. (1991). Manual for the Youth Self Report and 1991 profile. Burlington: University of Vermont.

Baron, R., y Kenny, D. (1986). The moderator-mediator variable distinction in social psychological research: Conceptual, strategic, and statistical considerations. Journal of Personality and Social Psychology, 51, 1173-1182.

Barsky, A. J., Peekna, H. M., y Borus, J. F. (2001). Somatic Symptom Reporting in Women and Men. Journal of General Internal Medicine, 16, 266275.

Cosgaya, L., Nolte, M., Martinez-Pampliega, A., Sanz, M. E., y Iraurgi, I. (2008). Conflicto interparental, relaciones padres-hijos e impacto emocional en los hijos. Revista de Psicología Social, 23, 29-40.

Coughlin Della Selva, P. (2006). Emotional processing in the treatment of psychosomatic disorders. Journal of Clinical Psychology, 62, 539-550.

Davidsdottir, S. (2006). Psychological Processes and Lifestyle by Age: Predictors for Psychosomatic Complaints. Counseling \& Clinical Psychology Journal, 3, 137-147.

Fava, G. A., y Sonino, N. (2005). The Clinical Domains of Psychosomatic Medicine. Journal of Clinical Psychiatry, 66, 849-858.

Frazier, P., Barron, K., y Tix, A. (2004). Testing Moderator and Mediator Effects in Counseling Psychology Research. Journal of Counseling Psychology, 51, 115-134.

García, M., \& Peralbo, M. (2000). Cultura, aculturación y percepción de las relaciones familiares. Infancia y Aprendizaje, 89, 81-101.

Holahan, C. J., y Moos, R. H. (1986). Personality, Coping, and Family Resources in Stress Resistance: 
A Longitudinal Analysis. Journal of Personality and Social Psychology, 5, 389-395.

Horner, K. L. (2001). Personality and intimate support influences on prospective health status. Psychology, Health \& Medicine, 6, 473-479.

Koerner, A., y Fitzpatrick, M. A. (2002a). Toward a Theory of Family Communication. Communication Theory, 12, 70-91.

Koerner, A., y Fitzpatrick, M. A. (2002b). Understanding Family Communication Patterns and Family Functioning: The Roles of Conver-sation Orientation and Conformity Orientation. Communication Yearbook, 26, 36-68.

Koerner, A., y Fitzpatrick, M. A. (2004). Communication in Intact Families. In A. Vangelisti (Ed.), Hanbook of Family Communication (pp. 177-195). Mahwah, New Jersey: Lawrence Erlbaum.

Kouneski, E. (2000). The family circumplex model, FACES II and FACES III: Overview of research and applications. Obtenido en Noviembre 27, 2008, de Universidad de Minessota, http://www. facesiv.com/pdf/faces_and_circumplex.pdf

McVey, J. M. (2002). Psychosomatic illness and anxiety in children: Their perceptions of family function and of physical pain. Texas: University of Texas at Austin.

Minuchin, S. (1975). A Conceptual Model of Psychosomatic Illness in Children: Family Organization and Family Therapy. Archives of General Psychiatry, 32, 116- 127.

Minuchin, S., Rosman, B., Baker, L., y Liebman, R. (1978). Psychosomatic Families. Cambridg: Harvard University Press.

Ochs, M., Seemann, H., Franck, G., Wredenhagen, N., Verres, R., y Schweitzer, J. (2005). Primary Headache in Children and Adolescents: Therapy Outcome and Changes in Family Interaction Patterns. Families, Systems \& Health: The Journal of Collaborative Family HealthCare, 23, 30-53.

Olson, D. H. (2000). Circumplex Model of Marital and Family Systems. Journal of Family Therapy, 22, 144-167.

Olson, D. H., y Gorall, D. M. (2003). Circumplex model of marital and family systems. En F. Walsh (Ed.), Normal Family Processes ( $3^{\circ}$ ed., pp. 514547). New York: Guilford. Olson, Russell y Sprenkle 1983.
Olson, D. H., Gorall, D. M., y Tiesel, J. W. (2006). FACES IV. Package. Administration Manual. Unpublished manuscript.

Olson, D. H., Sprenkle, D. H., y Russell, C. S. (1979). Circumplex model of marital and family systems, I: Cohesion and adaptability dimensions, family types, and clinical applications. Family Process, 18, 3-28.

Olson, D. H., Stewart, K. L., y Wilson, L. R. (1990). Health and stress profile (HSP), revised. Minneapolis: Profile of Health Systems.

Onnis, L. (1997). La palabra del cuerpo. Barcelona: Herder.

Peterson, C., Vaillant, G. E., y Seligman, M. E. P. (1988). Pessimistic Explanatory Style Is a Risk Factor for Physical Illness: A Thirty-Five-Year Longitudinal Study. Journal of Personality and Social Psychology, 55, 23-27.

Rivero, N., y Martínez-Pampliega, A. (2010). Adaptación cultural del Instrumento "Patrones de Comunicación Familiar- R". European Journal of Education and Psychology, 3, 141-153.

Rivero, N., Martínez-Pampliega, A., y Olson, D. (2010). Spanish Adaptation of the FACES IV Questionnaire. Psychometric Characteristics. The Family Journal, 18, 288-296.

Sanz, M., Iraurgi, I., y Martínez-Pampliega, A. (2002). Evaluación del funcionamiento familiar en toxicomanías: Adaptación española y características de adecuación métrica del FAP-FACES IV. En I. Iraurgi y F. González-Saiz (Eds.), Instrumentos de evaluación en drogodependencias (pp. 403434). Madrid: Aula Médica.

Sayre, G. G. (2001). The psychosomatic marriage: An empirical study. Tesis doctoral: Seattle Pacific University.

Schrodt, P., y Ledbetter, A. M. (2007). Communication Processes That Mediate Family Communication Patterns and Mental Well-Being: A Mean and Covariance Structures Analysis of Young Adults From Divorced and Nondivorced Families. Human Communication Research, 33, 330-356.

Segrin, C. (2006). Invited Article: Family Interactions and Well-Being: Integrative Perspectives. Journal of Family Communication, 6, 3-21.

Segrin, C., Badger, T., Sieger, A., Meek, P., y Lopez, 
A. M. (2006). Interpersonal well-being and mental health among male partners of women with breast cancer. Issues in Mental Health Nursing, 27, 371389.

Tamada, T. (2005). The influence of sex differences and social environment on the occurrence of psychosomatic disorders. Journal of Psychosomatic Obstetrics \& Gynecology, 26, 1-1.
Taylor, S. (1991). Health Psychology. New York: Mc Graw Hill.

Terre, L., y Ghiselli, W. (1997). A developmental perspective on family risk factors in somatization. Journal of Psychosomatic Research, 4, 197-208.

Zikmund, V. (1992). Health and Disease: Biological, Psychological and Social Aspects. Studia Psychologica, 34, 101-113.

Manuscrito recibido: 09/02/2010

Revisión recibida: 20/05/2010

Aceptado: 21/03/2011 